\title{
Materials affecting neonatal and environmental health
}

\author{
A. Marshall-Baker \\ Department of Interior Architecture, \\ University of North Carolina at Greensboro, USA
}

\begin{abstract}
Industrial activity provides products of service and convenience that perhaps unintentionally compromise human and environmental health. Yet these products and materials are ubiquitous, even in those environments intended to foster wellness. Persistent bioaccumulative toxins and volatile organic compounds are two categories of substances that consistently appear in interior products and materials, and are associated with temporary conditions such as eye irritation, chronic conditions such as chemical sensitivity, and terminal conditions such as cancer. Identifying and developing healthful alternative materials is facilitated by advancing technologies, including nanoscience, innovative approaches, e.g., biomimicry, and comprehensive efforts that consider environmental, equitable, and economic impacts such as the Joint Committee for Sustainable Textiles.

Keywords: sustainability, green materials, healthcare, interior environments, persistent bioaccumulative toxins, volatile organic compounds, newborn intensive care.
\end{abstract}

\section{Introduction}

An intention of industrial activity throughout the $20^{\text {th }}$ century was the manufacture of materials and substances that seemingly made our lives better in various ways. Mass production provided a wide range of products that generally were less expensive, manufactured in large quantities, readily available, ubiquitous, continuously upgraded, and easily replaced - and they still are. Yet an unintended consequence of this activity was enormous albeit nonobvious costs to soil, air, water, and general well-being. Some of these products and materials, for example, contain substances that are harmful to human health such 
as formaldehyde. Other products and materials require exploitation of individuals who comprise the work force necessary to bring the product to bear. Development of other products and materials drives the sacrifice of one source of livelihood for another, such as tourism for mining. And most materials and substances degrade the air, soil, or water at some point in their life cycle perhaps during the harvest of the raw materials or at the end of their useful life when the products are relegated to a landfill or an incinerator. These all are issues that preclude the use or development of materials that are "green" or sustainable. Other than products of nature that are truly cyclic across their lifespans as described in the cradle to cradle protocol [1], most products are not sustainable and many are harmful to human and environmental health. This is the current condition even in healthcare settings, despite their intention to facilitate wellness. Concern about the environmental quality of interior environments is particularly salient to patients including neonates who are unable to manage exposure to harmful environmental agents. The purpose of this paper is to recognize substances harmful to human and environmental health that are typical of healthcare settings, identity feasible substitutes, and speculate alternative approaches and technological advances that may address the current concerns.

\section{Products and materials in healthcare environments}

At a conference in 2007 regarding newborn intensive care, Lynne Wilson-Orr, architect, designer, and principal at Parkin Architects in Toronto, described with colleagues in healthcare a typical hospital room with its usual installation of products and materials $[2,3]$. They articulated locations of materials that often treatments, and furnishings. For patients who are newborns, their typical hospital

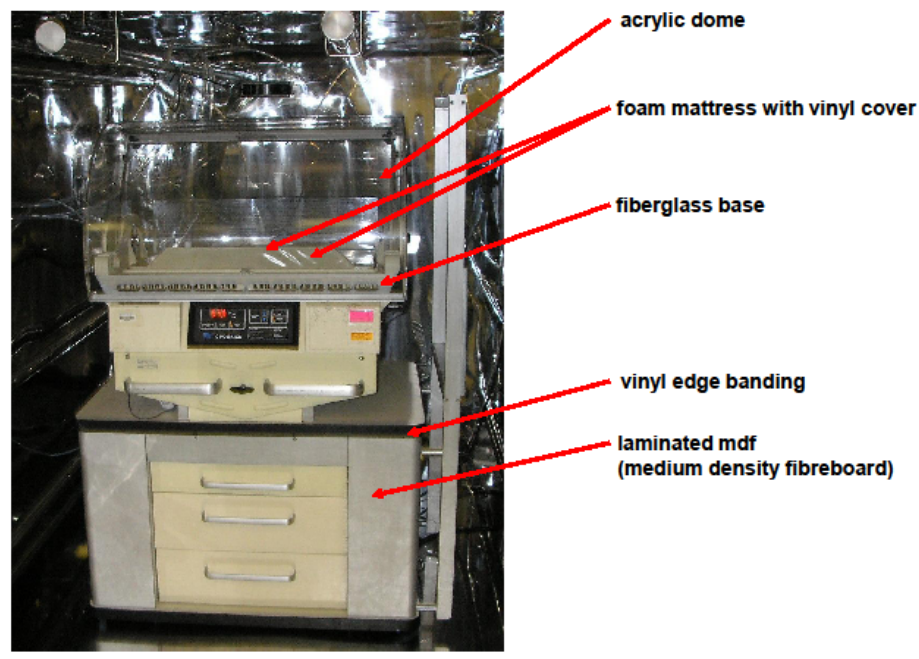

Figure 1: Materials that are typical of infant incubators, regardless of manufacturer. 
"room" is an incubator which also harbours harmful substances in the infant chamber, cabinetry, and mattress, as well as in caregiving materials such as enteral bags and intravenous tubing, fig.1. Knowledge of the effects of materials such as these on patients or occupants of interior space is critical to those involved in the design of the built environment. Developing or acquiring this knowledge begins with a fundamental understanding of the substances that are prevalent in products and materials readily available for installation, application, and use in our interior environments.

\section{Substances harmful to human and environmental health}

Two broad categories of indoor pollutants are persistent bioaccumulative toxins (PBTs) and volatile organic compounds (VOCs). Substances classified as either or both PBTs and VOCs may cause short term health conditions such as eye irritation, chronic conditions such as asthma, chemical sensitivity, and allergies, or terminal conditions such as cancer. PBTs and VOCs that cause these conditions may be absorbed through the skin, ingested, or inhaled, and all are pervasive in interior environments.

PBTs do not break down easily in the environment - they "persist." Thus, exposure over time results in an accumulation that magnifies up the food chain. For example, PBTs accumulate in large fish not only because of their individual exposure to PBTs, but also from the amounts of PBTs stored in the tissues of the smaller fish that the larger fish ingest - and that PBT count in the smaller fish accumulates as a consequence of the PBTs they ingest by eating smaller marine animals. Through this process, PBTs "bio-accumulate," thereby providing the highest concentrations to those animals at the top of the food chain: human beings. Heavy metals such as arsenic, lead, mercury, and cadmium all are PBTs and all are known to affect negatively the nervous system, liver and kidneys, and to be associated with developmental delays, lowered IQ scores, and cancer [4].

Once ingested, PBTs reside in the fatty tissues of animals but convey easily. A nursing mother, for example, transfers PBTs to her infant through breast milk [5]. Because the detrimental effects of PBTs on human and environmental health are well-known, organizations such as the U.S. Environmental Protection Agency (USEPA) establish specific levels of exposure to toxic substances, e.g., heavy metals. A consequence often is a recommendation such as that from the USEPA to limit consumption of large fish including tuna. Recent reports, however, indicate that even trace amounts of harmful substances may have significant health effects [6].

Some PBTs are also volatile organic compounds (VOCs) which are measured as organic gasses [7]. VOCs are the primary source of indoor air pollution. The USEPA reports that levels of VOCs average 2-5 times higher in indoor than outdoor environments though Greenpeace reports that these levels are 100 times higher. Health effects of VOCs are directly related to the amount of exposure, but again can range from irritation to allergies to chemical sensitivity to cancer.

Polyvinyl chloride (PVC) is perhaps the most pervasive VOC in interior environments. It is in our kitchen utensils, the paint on the walls, and the 
plumbing in our homes. It is in children's toys, shower curtains, and covers of 3ring binders. It insulates wires in our computers, covers our floors, and binds the backs of our carpets and rugs. Though the dangers of PVC are seemingly invisible because they occur "upstream" in its manufacture or "downstream" in its disposal (in a landfill or incinerator), the use of PVC also is dangerous [8]. PVC is made from chlorine and ethylene. Chlorine is typically the product of a mercury cell process which is a significant source of mercury pollution. When chlorine and ethylene combine, two intermediate chemicals result: ethylene dichloride which is classified as a possible human carcinogen and vinyl chloride monomer which is a known carcinogen. The consequence of these two combined chemicals is polyvinyl chloride or PVC.

PVC is not only made from two carcinogenic substances, but also releases dioxins as a by-product of the manufacturing process. Dioxins also are classified as carcinogenic as well as reproductive and developmental toxicants which alter the immune and endocrine systems. Dioxins were considered a larger environmental and health problem when most hospital waste was incinerated and dioxins were released into the air. Although that practice has been greatly reduced, much of the presumed nonhazardous waste from hospitals such as iv bags and tubing still may be incinerated, and these supposed benign products do release dioxins into the air during incineration. Wilson-Orr and colleagues [2] report that 80 million tons of PVC are used in hospitals each year.

PVC is an example of a product of industrial activity that is inexpensive, manufactured in large quantities, readily available, ubiquitous, and thus easily replaced. It is generally irreparable and typically ends its one useful life or purpose in a landfill or incinerator. Other costly PBTs and VOCs include:

- Phthalates. PVC is brittle and hard when it is first produced. Phthalates or "plasticizers" such as di-2-ethylhexyl-phthalate (DEHP) are additives that soften PVC, making it more flexible and pliable. Catheters, IV bags and tubing, and enteral nutrition feeding bags typically are made with PVC and DEHP. Nurses in newborn intensive care first became aware of a problem with plasticizers when PVC tubing inserted into preterm infants lost its suppleness, presenting as a harder more brittle material when it was removed from the infants. This change in texture indicated that the plasticizer had leached into the infant [2]. DEHP is a VOC that impairs reproductive health, particularly in infant boys, and is reported to suppress the immune system and damage organs and the nervous system $[9,10]$.

- Polychlorinated biphenyls (PCBs) are chemical mixtures that are nonflammable and chemically stable with high-insulating properties [11]. These qualities make PCBs ideal for use in materials such as paint, plastics, and rubber products. But PCBs are PBTs that accumulate to levels that are carcinogenic. 
- Brominated or halogenated flame retardants (BRFs) such as polybrominated diphenyl ethers (PBDEs) are used to reduce the risk of fire in furniture, textiles, and electronic equipment. BRFs are PBTS and suspected endocrine disruptors that produce dioxins and furans when heated. They typically are found on clothing and blankets such as infant sleepers and bed linens [12].

- Perfluorochemicals (PFCs) make products resistant to heat, oil, stains, grease and water [13]. PFCs are VOCs and typically used in nonstick products and stain-resistant carpets and fabrics such as window treatments and upholstery.

- Urea formaldehyde is a VOC that is toxic, allergenic, and considered a probable carcinogen by the USEPA [14]. It is used as a binder in medium density fibreboard (mdf) and in fibreglass to reduce the selfabrasive qualities of the individual filaments that foster breaking. Urea formaldehyde foam is used as an insulating material and in furnishings, including upholstery foam, pillows, and mattress pads. Formaldehyde also is used as a binder in adhesives, a preservative in paint, and can add permanent press qualities to clothing and drapery.

- Acrylic, a clear plastic, is a product of acrylic acid which is classified as a VOC. Though not considered carcinogenic by the USEPA, acrylic acid is known to be an eye, skin, and mucous membrane irritant [15]. In addition to plastics, products of acrylic acid include floor polish, coatings or finishes for upholstery, paint, and the infant chamber on the incubator.

Despite their toxic qualities, all of these substances are typical of our day to day environments as well as our healthcare environments. The materials used in healthcare such as ceiling tiles, floor and wall coverings, window treatments, millwork, and furnishings likely contain PVC, acrylics, formaldehyde, phthalates, perfluorochemicals, and brominated flame retardants. These same substances likely occur in the acrylic dome of the infant incubator, its mattress and vinyl cover, the fibreglass base, and mdf cabinet, despite an intention to provide a controlled, healthful environment for newborn infants whose health is compromised by a variety of conditions or events. When we tested the air quality of an infant incubator that was 8 years-old, for example, a series of toxic substances appeared in the analysis as shown in table 1 [3]*. Of the 20 individual chemicals emitting into the air during a 24 hour testing period, some such as cinnamaldehyde appear to be scents in cleaning agents that dissipated rapidly. Others such as formaldehyde and acetaldehyde, known carcinogens, had a constant presence during testing that may have emanated from the fibreglass base, vinyl covered foam mattress, or mdf cabinet. The levels of these two substances measured twice that recommended by the state of California. Importantly, recommended levels of exposure typically are calculated for adults, not children and certainly not developing infants whose arrival in an extrauterine 


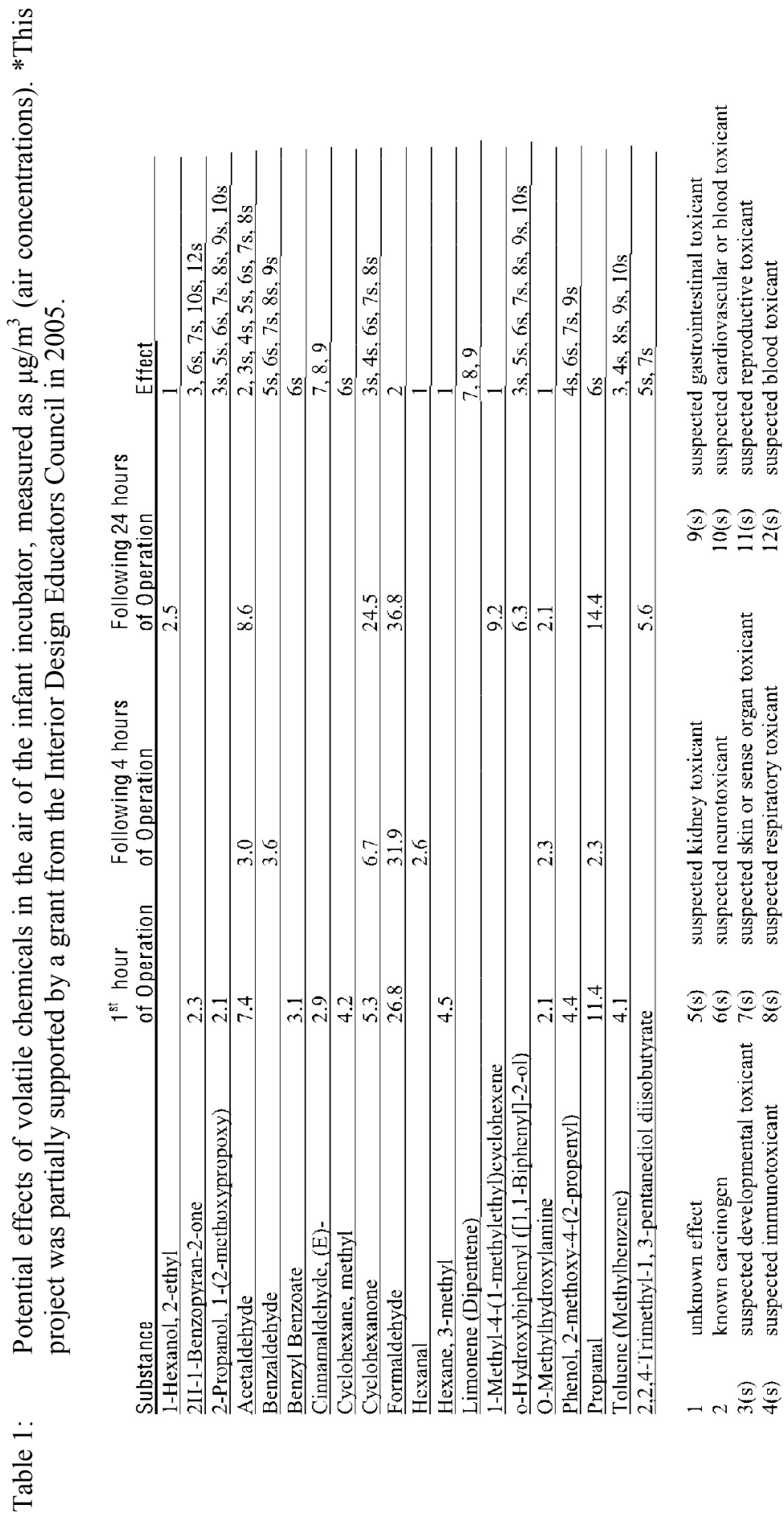


environment may have been premature. Thirteen other toxicants present in the incubator air were known or suspected toxicants affecting systems including respiratory, gastrointestinal, and reproductive as shown in table 1.

\section{Alternative materials}

In many instances, alternative materials can be used that are not - or at least may be less - harmful to human health. For example, linoleum and rubber flooring can be used in healthcare environments instead of sheet vinyl. Carpets backed with chlorine-free and PVC-free materials such as polyvinyl buteral or thermoplastic that are installed using low- or no-VOC adhesives reduce or eliminate VOCs in the indoor environment [16]. PVC- and fibreglass-free wall coverings are more difficult to find, although no-VOC paint is readily available. Formaldehyde-free composite woods and solid polymer counter tops can substitute for laminated cabinets and counters. Furnishings made of steel, polypropylene, polyethylene, and wood can be used instead of PVC, acrylics, and other plastics. Soy-based foam is an alternative to urea formaldehyde foam. Fabric backed or moulded chairs can preclude the use of upholstery foam altogether, and selecting fabrics that are inherently stain resistant such as polyester eliminates the need for finishes such as brominated flame retardants. Rubber, polyethylene, or nylon membranes can replace vinyl mattress covers.

Regarding medical equipment, polyethylene and silicone are safer alternatives for tubing and enteral feeding tubes that typically are made from PVC and DEHP. Intravenous products may be made from polypropylene or polyethylene, materials that can be reclaimed and recycled. Glass is a substitute for some acrylic applications. Yet although these alternative materials, e.g., linoleum, are safer to human health than more typical products such as vinyl floor covering, not all of these products are considered ecologically safe.

\section{Needed substitutes}

Alternative materials such as nylon, polyester, polyethylene, and polypropylene may not contain PBTs and may be low- or no-VOC products and materials, but they still may be harmful. Each of these materials relies on petroleum. Nylon fibre used in carpets and fabrics, for example, is extruded from petroleum. It may not be a PBT or a VOC and it ultimately may be reclaimed and reused in a technical cycle described by the cradle to cradle protocol [1], but it relies on fossil fuels. This is an enduring problem regarding "green" or sustainable materials. So often there is not an alternative material that is sustainable throughout its life cycle, from harvest to process, through packaging and shipping, installation, use, and maintenance, then reclamation, reuse, or disposal. Beyond the environmental effects are economic and equitable considerations regarding the sustainable nature of a product. If the work force necessary to bring a product to market is not paid a living wage, then the product, despite its "green" qualities, is not sustainable because the work force necessary for its production is not sustainable. If the product is a consequence of an economic 
trade-off such as compromising eco-tourism for mountain-top removal, then the product's economic impact is not sustainable and, thus, neither is the product sustainable. These are challenges that manufacturers must address.

\section{Promise of new technologies and approaches}

Almost daily, new technologies and approaches surface to address critical, global needs related to development of sustainable products and practices that support human and environmental health. Although development of truly sustainable products and practices remains complicated and complex, new directions of industrial activity that include nanoscience, closer looks at nature, and comprehensive approaches offer great promise for the immediate future.

Reyad Sawafta uses nanoscience and nanoengineering to develop products that are less costly to human and environmental health [17]. For example, Sawafta developed a drywall material that holds and releases heat, thereby reducing the amounts of fossil fuels necessary to heat a space. Incorporating into drywall phase change materials that store the energy they generate when changing from a solid to a gel enables the wall or ceiling surface of a room to absorb, store, and then release heat across the course of a day. In a separate project, Sawafta is using nanoarchitecture to develop diapers and other products intended to absorb moisture that either maintain or collapse their structure when wet. The diaper absorbs and holds moisture from an infant, for example, who is in either a supine or prone position. Yet if that diaper is then placed in a toilet, the nanoarchitecture that preserved the structure of the diaper when moisture was absorbed in specific ways begins to deconstruct. Because of the onslaught of moisture from all directions, the diaper degrades to the point that it may be flushed down the toilet rather than sent to a landfill.

Janine Benyus extols scientists and industrialists to study the ways and means of nature through biomimicry $[18,19]$. She reminds us, for example, that spiders spin a fibre that is proportionally stronger than steel and is the consequence of "eating dead flies." She reminds us that barnacles produce an adhesive as strong or stronger than that produced using the best synthetic substances available, and that barnacles produce this adhesive under water. She reminds us that the feathers of peacocks do not include iridescent blue pigments, but rather a physical structure which bends light rays that we then perceive as "blue." Through workshops, seminars, and lectures, Benyus encourages thinking that models the effective processes of nature. As a consequence of this approach, more and more products and materials that mimic the healthful practices of nature are being developed such as structural paint which is based on the principle of bending light rays rather than adding pigments; self-cleaning paint that "rolls" drops of water down its surface collecting dirt and debris similarly to that of rain rolling off of leaves; and "gecko tape" that mimics the small vacuum spaces that geckos create with their feet that hold surfaces together without the use of adhesives.

Unfortunately, not all these technological processes using nanoscience and biomimicry are themselves sustainable. Nanoarchitecture, for example, can 
produce "nano" waste. Yet many manufacturers are looking toward renewable sources for materials. Plastics, for example, can be biodegradable when they are made from corn [20]. Soy-based foam can replace urea formaldehyde foam. Sugar cane rather than petroleum is being used to produce green polyethylene. Even in these instances, however, the potential for a secondary problem exists when food crops are diverted to industrial use. This is one concern surrounding ethanol made from corn. Instead of food crops, agricultural waste is sometimes used as a renewable source that does not compromise the food supply. Wheatboard or strawboard, for example, is a material often used in cabinetry that is made from the shafts of wheat. Kirei board is made from sorghum straw and can be used in cabinetry and furniture.

One last example regarding green products and materials is development of a sustainable standard for commercial textiles. This is an on-going effort by a multinational Joint Committee for Sustainable Textiles coordinated by the National Standards Foundation. This standard considers environmental, equitable, and economic impacts throughout the supply chain of commercial fabrics including raw materials, suppliers, manufacturers, purchasers, fabricators, and users. The intent of the sustainable standard is not only to measure existing products and practice, but to set benchmarks for continued improvement and innovation.

\section{Conclusion}

Altering traditional or standardized use of materials is difficult not only because of the knowledge necessary to do so, but also because appropriate substitutes may not exist. Yet becoming knowledgeable about materials and viable substitutes is critical to designers and others who select and specify materials for our indoor environments. This is particularly important to individuals in healthcare settings who not only anticipate wellness, but who also are not positioned to affect their exposure to harmful substances. Designers and others responsible for the built environment shoulder this responsibility on behalf of the occupants of the space. Fortunately, global awareness and demand for more healthful products and practices is increasing and industries are responding with advancing technologies such as nanoscience, alternative approaches such as biomimicry, and comprehensive efforts such as the work of the Joint Committee for Sustainable Textiles.

\section{References}

[1] McDonough, W. \& Braungart, M. Cradle to Cradle: Remaking the Way We Make Things, North Point Press: New York, 2002.

[2] Wilson-Orr, L., Archer, J., MacMillan-York, E., MacIsaac, E., \& Reid, A., Greening of a nursery. Syllabus of the $20^{\text {th }}$ Annual Gravens Conference on the Physical and Developmental Environment of the High Risk Infant: Clearwater Beach, Florida, (no page number), 2007.

[3] Marshall-Baker, A. The design of neonatal intensive care units: Information gathering and practitioners as consumers of research (Chapter 6). Informing 
Design, eds. J. Dickinson \& J.P. Marsden, Fairchild Books: New York, pp. 159-190, 2009.

[4] Zadorozhnaja, T.D., Little, R.E., Miller, R.K., Mendel, N.A., Taylor, R.J., Presley, B.J., \& Gladen, B.C. Concentrations of arsenic, cadmium, copper, lead, mercury, and zinc in human placentas from two cities in Ukraine. Journal of Toxicology and Environmental Health, 61, pp. 255-263, 2000.

[5] United States Environmental Protection Agency (USEPA). Pollution Prevention and Toxics Website, Washington DC, http://www.epa.gov/ opptintr/index.htm

[6] Waldman, P. Common industrial chemicals in tiny doses raise health issue. The Wall Street Journal, A1, 25 July 2005.

[7] United States Environmental Protection Agency (USEPA). Indoor Air Quality Website, Washington DC, http://www.epa.gov/iaq/voc.html

[8] Health Care Without Harm, http://www.noharm.org/all_regions/issues/ toxins/pvc_phthalates/

[9] Calafat, A.M., Needham, L.L., Silva, M.J., \& Lambert, G. Exposure to di(2-ethylhexyl) phthalate among premature neonates in a neonatal intensive care unit, Pediatrics, 113(5), pp. e429-e434, 2004.

[10] Swan, S.H., Main, K.M., Liu, F., Stewart, S.L., Kruse, R.L., Calafat, A.M., Mao, C.S., Redmon, J.B., Ternand, C.L., Sullivan, S., Teague, J.L., and the Study for Future Families research Team. Decrease in anogenital distance among male infants with prenatal phthalate exposure, Environmental Health Perspectives, 113(8), (no page number), 2005.

[11] United States Environmental Protection Agency (USEPA). Polychlorinated biphenyls Website, Washington DC, http://www.epa.gov/osw/hazard/ $\mathrm{tsd} / \mathrm{pcbs} /$ index.htm

[12] Darnerud, P.O. Toxic effects of brominated flame retardants in man and wildlife, Environment International, 29, pp. 841-853, 2003.

[13] Minnesota Department of Health. Perfluorochemicals Website, http://www.health.state.mn.us/divs/eh/hazardous/topics/pfcshealth.html

[14] United States Environmental Protection Agency (USEPA). Indoor Air Quality Website, Washington DC, http://www.epa.gov/iaq/formalde.html

[15] United States Environmental Protection Agency (USEPA). Technology Transfer Network Air Toxics Website, Washington DC http://www.epa.gov/ttn/atw/hlthef/acrylica.html

[16] Health Care Without Harm, http://www.premierinc.com/qualitysafety/tools-services/safety/topics/epp/downloads/hcwh-alternatives-pvcdehp.pdf

[17] QuarTek, http://www.quartekcorp.com/

[18] Benyus, L. Biomimicry: Innovation inspired by nature, New York: HarperCollins Publishers Inc.

[19] Ask Nature, http://www.asknature.org/

[20] NatureWorks LLC, http://www.natureworksllc.com/ 